\title{
A Simplified Accuracy Enhancement to the Saleh AM/AM Modeling and Linearization of Solid-State RF Power Amplifiers
}

\author{
Haider Al-Kanan *(D) and Fu Li * \\ Department of Electrical and Computer Engineering, Portland State University, Portland, OR 97207-0752, USA \\ * Correspondence: alkanan.haider@gmail.com (H.A.); lif@pdx.edu (F.L.)
}

Received: 8 October 2020; Accepted: 29 October 2020; Published: 31 October 2020

\begin{abstract}
The Saleh behavioral model exhibits high prediction accuracy for nonlinearity of traveling-wave tube power amplifiers (TWT-PAs). However, the accuracy of the Saleh model degrades when modeling solid-state power amplifiers (SSPAs) technology. In addition, the polynomial expansion of the Saleh model consists of only odd-order terms as analyzed in this work. This paper proposes a novel model accuracy enhancement for the Saleh amplitude-to-amplitude (AM/AM) model when applied to radio frequency (RF) SSPAs. The proposed model enhancement accounts for the second-order intermodulation distortion, which is an important nonlinearity challenge in wideband wireless communications. The proposed static AM/AM model is a three-parameter rational function, which exhibits low complexity compared to the state-of-the-art behavioral models. A transpose architecture of finite-impulse digital filter is used to quantify the memory effect in SSPAs. A least-squares method is used for extracting all the model parameters. A linearization technique using a three-parameter digital predistortion model is also calculated to compensate for the AM/AM nonlinear distortion in SSPAs. Finally, the identification and evaluation of the enhanced Saleh model is presented based on measurements of RF SSPAs.
\end{abstract}

Keywords: solid-state power amplifiers; behavioral models; digital predistortion; nonlinear distortion; memory estimation

\section{Introduction}

The rapid evolution of wireless communications requires high dynamic range RF power amplifiers (PAs) to efficiently operate on high-amplitude fluctuations in modern digital modulation [1]. Solid-state power amplifiers (SSPAs) are intensively deployed devices in the front ends of mobile systems [2-4]. Therefore, empirical models for SSPAs have been utilized for decades to study the distortion effects on wireless communications systems.

Accurate and low-complexity behavioral models for SSPAs have become important techniques in simulating and linearizing communication systems [5-7]. In particular, an accurate estimation of the model smoothness in the amplitude-to-amplitude (AM/AM) transition from the linear to the saturation regions has been a topic of interest in the state-of-the-art PA modeling [8-12]. This is because operating SSPAs near the compression region is often required for achieving high power efficiency and transmitting signals of high peak-to-average power ratio (PAPR) [13].

Rapp, Ghorbani, and Cann behavioral models are popular mathematical functions that match well the AM/AM characteristics of SSPAs. However, these models often consist of complicated rational functions; therefore, starting points and iterative estimation methods are typically required for such model estimation. In addition, these models exhibit a lack of relevance in modeling digital predistortion (DPD) for linearizing SSPAs. 
The polynomial-based models are commonly used for SSPAs, because polynomials provide sufficient modeling flexibility in estimating the parameters and adjusting the model accuracy. However, polynomial models are subject to estimation errors such as curve under-fitting and over-fitting model prediction when truncated nonlinear coefficients are employed.

The AM/AM characteristics of traveling-wave tube power amplifiers (TWT-PAs) exhibit high nonlinearity and roll-over effects near the saturation region. Thus, the accuracy of the Saleh model significantly degrades when modeling the compression smoothness of the SSPAs. The proposed modified Saleh model by O'Droma achieves substantial accuracy improvement for modeling the AM/AM nonlinearity for laterally-diffused metal-oxide semiconductor (LDMOS) SSPAs [9]. However, O'Droma proposed a six-parameter rational formula, which is computationally expensive. In addition, the direct inversion of the O'Droma model is mathematically difficult for calculating the predistortion model. Hence, this paper presents a simplified behavioral model with an adequate AM/AM accuracy improvement for SSPA technologies.

The amplitude-to-phase distortion is often minor in SSPAs and is assumed insignificant in this simplified modeling approach [10]. The presented modeling approach consists of three parameters, which allows better nonlinearity modeling for a large signal amplitude beyond the 1-dB compression point. In addition, the presented expression is convenient to characterize the even-order intermodulation distortion (IMD) for wideband and multiband communications.

A simplified and low complexity DPD model is also calculated to compensate for the AM/AM nonlinearity in SSPAs. This paper is organized as follows: Section 2 describes the proposed enhancement approach to the Saleh AM/AM model, followed by an approach to characterize the dynamic memory effect in Section 3. Section 4 presents a linearization model for SSPA using DPD. Section 5 includes the experiment and modeling results for both the SSPA and DPD model. Finally, conclusions are presented in Section 6.

\section{Enhanced Approach for SSPAs}

The Saleh AM/AM model is a two-parameter function. The first parameter $\alpha$ represents the small signal gain, and the second parameter $\beta$ is used to adjust the transition sharpness (i.e., gain compression) from the linear region to saturation region. The AM/AM Saleh model $\mathrm{F}[u(\mathrm{t})]$ can be expressed as [8]:

$$
\mathrm{F}[u(t)]=\frac{\alpha u(t)}{1+(\sqrt{\beta} u(t))^{2}}
$$

where $u(t)$ is the envelope of the PA input baseband signal. The Saleh model is an odd function $\{\mathrm{F}[-u(\mathrm{t})]$ $=-\mathrm{F}[u(t)]\}$, and the polynomial expansion of the rational function in Equation (1) is given by:

$$
\mathrm{F}[u(t)]=\sum_{k=0}^{\infty}(-1)^{k} \alpha \beta^{k} u^{(2 k+1)}(t)
$$

where $k$ is an integer number $\{k=0,1,2,3, \ldots \ldots\}$

$$
\mathrm{F}[u(t)]=\alpha u(t)-\alpha \beta u^{3}(t)+\alpha \beta^{2} u^{5}(t)+\ldots \ldots
$$

The polynomial series in Equation (3) consists of the nonlinear coefficients $\left\{\alpha, \alpha \beta, \ldots, \alpha \beta^{k}, \ldots\right\}$. Equation (3) shows a mathematical relationship of a magnitude-dependency among all the polynomial coefficients. In comparison with the typical odd-order Taylor model in Equation (4), the Taylor coefficients are often considered statistically and linearly independent parameters. Therefore, the Taylor model exhibits a higher degree of freedom for fitting the polynomial coefficients to the observed 
data. However, the polynomial curve fitting is subject to uncertainty and often exhibits high prediction errors beyond the range of the observed data.

$$
\mathrm{T}[u(t)]=c_{1} u(t)+c_{3} u^{3}(t)+c_{5} u^{5}(t)+\ldots \ldots
$$

The magnitude of the Taylor coefficients $\left\{c_{1}, c_{3}, \ldots \ldots\right\}$ are considered device-dependent, but the independent coefficients are insufficient for describing the effect of the higher order IMDs on the lower order IMDs. For example, the fifth-order IMD affects the third-order IMD and so on [14]. The higher order Taylor polynomial can achieve better model accuracy to fit the nonlinear curvature of the AM/AM conversion, because using additional independent coefficients increases modeling the dimensions of the nonlinear system, but this often leads to a noisy estimate and poor coverage of confidence intervals.

The typical output of the AM/AM Saleh model increases almost linearly in the small signal magnitude at the rate $\alpha$. However, the gain of the Saleh model decreases monotonically after the maximum curvature (this occurs at $|u|=\beta^{-1 / 2}$ ) [15] and approaches zero for very large theoretical values of $|u|$. This is one of the main weaknesses in the Saleh model when applied to SSPAs, because the saturation level in SSPAs is almost constant and specified by the supply voltage on the drain/collector of the output transistor. Power amplifiers are typically designed to operate over a dynamic range of the load-line with the DC quiescent point "Q-point" which is set in the active region of the transistor on a small amplitude signal (e.g., Q-point is set to the middle of the load-line in a class-A design). However, on a large input amplitude signal, the output signal becomes extensively large and forces the output signal to surpass the power supply voltage and becomes flattened. Therefore, the output signal is limited by the supply voltage in the saturation region [16].

A simple model enhancement is introduced in this work to address the model limitations by including an additional quadratic term within the numerator of the Saleh model [17] as illustrated

$$
\mathrm{F}_{\mathrm{es}}[u(t)]=\frac{\alpha u(t)+\lambda u^{2}(t)}{1+\beta u^{2}(t)}
$$

where $\mathrm{F}_{\mathrm{es}}[u(t)]$ is the proposed enhanced Saleh AM/AM model. The parameter $\lambda$ is a real positive number introduced here for adjusting both gain and roll-over near the saturation region. The small signal gain $\alpha$ of the model in Equation (5) is approximately equal to the linear gain of the Saleh model as depicted:

$$
\lim _{u \rightarrow 0} \frac{\partial\left(\mathrm{F}_{\mathrm{es}}[u]\right)}{\partial(u)}=\alpha
$$

The parameter $\lambda$ causes the function in Equation (5) to be mathematically non-monotonic and the output increases asymptotically toward the saturation for a large signal amplitude (saturation level $\approx$ $\lambda / \beta$ ). Finally, the polynomial expansion of Equation (5) becomes a mixed nonlinear combination of odd and even terms as follows:

$$
\begin{gathered}
\mathrm{F}_{\mathrm{es}}[u(t)]=\sum_{k=0}^{\infty}(-1)^{k} \beta^{k} u^{(2 k)}(t)\left(\alpha u(t)+\lambda u^{2}(t)\right) \\
\mathrm{F}_{\mathrm{es}}[u(t)]=\alpha u(t)+\lambda u^{2}(t)-\alpha \beta u^{3}(t)-\lambda \beta u^{4}(t)+\alpha \beta^{2} u^{5}(t)+\lambda \beta^{2} u^{6}(t)-\alpha \beta^{3} u^{7}(t)+\ldots . .
\end{gathered}
$$

Equation (8) illustrates that the magnitude of the second-order IMD is completely characterized by the parameter $\lambda$. The other higher order even-terms in this model are characterized by the nonlinear coefficients $\{\lambda, \lambda \beta, \ldots, \lambda \beta k, \ldots\}$. In addition, the proposed model enhancement can improve the flatness in the saturation region, because the mathematical expression in Equation (8) converges asymptotically to the constant value $(\lambda / \beta)[17]$.

The second-order IMD of SSPAs is an ongoing concern in wideband and multiband communications $[14,18,19]$. Figure 1 shows the typical two-tone IMDs, which illustrates the locations 
of the second-order distortion $\left(\mathrm{f}_{2}-\mathrm{f}_{1}, \mathrm{f}_{2}+\mathrm{f}_{1}\right)$ with respect to the fundamental tones for wideband communications [14].

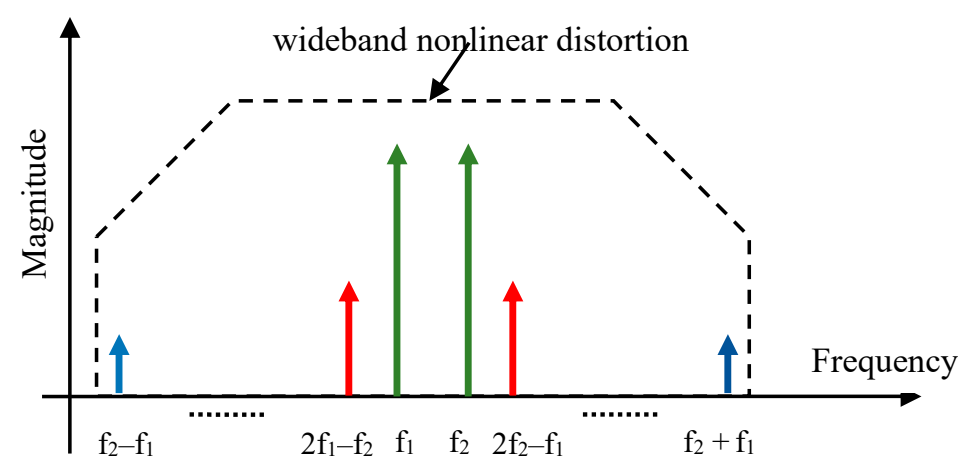

Figure 1. Wideband output spectrum of RF power amplifier illustrating the two-tone harmonic distortion effect.

\section{Model Estimation}

A least-squares method is used for calculating the parameters of the enhanced Saleh model as described in this section. Substituting $z(t)=\mathrm{F}_{\mathrm{es}}[u(t)]$ in Equation (5) and re-arranging the equation variables results in

$$
z(t)=\alpha u(t)+\lambda u^{2}(t)-z(t) \beta u^{2}(t)
$$

where $z(t)$ is the envelope of the PA baseband output signal. A matrix equation can be formulated from Equation (9) by substituting discrete-time samples of both $u(t)$ and $z(t)$ as illustrated

$$
\left[\begin{array}{c}
z(0) \\
z(1) \\
\vdots \\
z(n)
\end{array}\right]=\left[\begin{array}{ccc}
u(0) & -z(0) u^{2}(0) & u^{2}(0) \\
u(1) & -z(1) u^{2}(1) & u^{2}(1) \\
\vdots & \vdots & \vdots \\
u(n) & -z(n) u^{2}(n) & u^{2}(n)
\end{array}\right]\left[\begin{array}{c}
\alpha \\
\beta \\
\lambda
\end{array}\right]
$$

For simplicity, a matrix notation is used in representing (10)

$$
\mathbf{z}=\mathbf{U c}
$$

where $\mathbf{z}$ is a column vector of $((n+1) \times 1)$ elements, $\mathbf{c}$ is a column vector of the model parameters $(3 \times$ $1)$, and $\mathbf{U}$ is a matrix consisting of $((n+1) \times 3)$ elements of the input and output samples. Finally, the vector $\mathrm{c}$ is calculated as:

$$
\mathbf{c}=\left(\mathbf{U}^{\mathrm{T}} \mathbf{U}\right)^{-1} \mathbf{U}^{\mathrm{T}} \mathbf{z}
$$

where ()$^{\mathrm{T}}$ denotes the operator of a matrix transposition [17].

\section{Memory Modeling}

Memory effect is a popular hysteresis phenomenon in RF PAs which causes dynamic nonlinear dispersion in the AM/AM conversion [20,21]. Memory effect is considered device-dependent due to the impedances' variation of both the matching and biasing networks with respect to operating frequency, and to the temperature variation in transistors' junctions as depicted in Figure 2. 


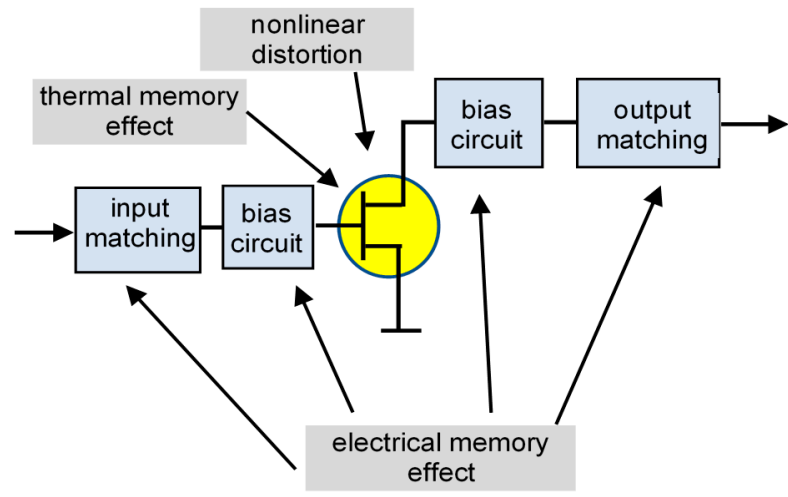

Figure 2. Simplified architecture of the memory component elements of the RF power amplifier.

A digital filter is a straightforward approach for modeling the memory effect in RF PAs. In this work a transpose finite-impulse response (FIR) digital filter is used in cascade with the enhanced Saleh model based on Hammerstein modeling theory as depicted in Figure 3. The architecture of the transpose FIR filter is computationally less complex than the direct FIR filter in real-time implementation because it supports multiple constant multiplication technique.

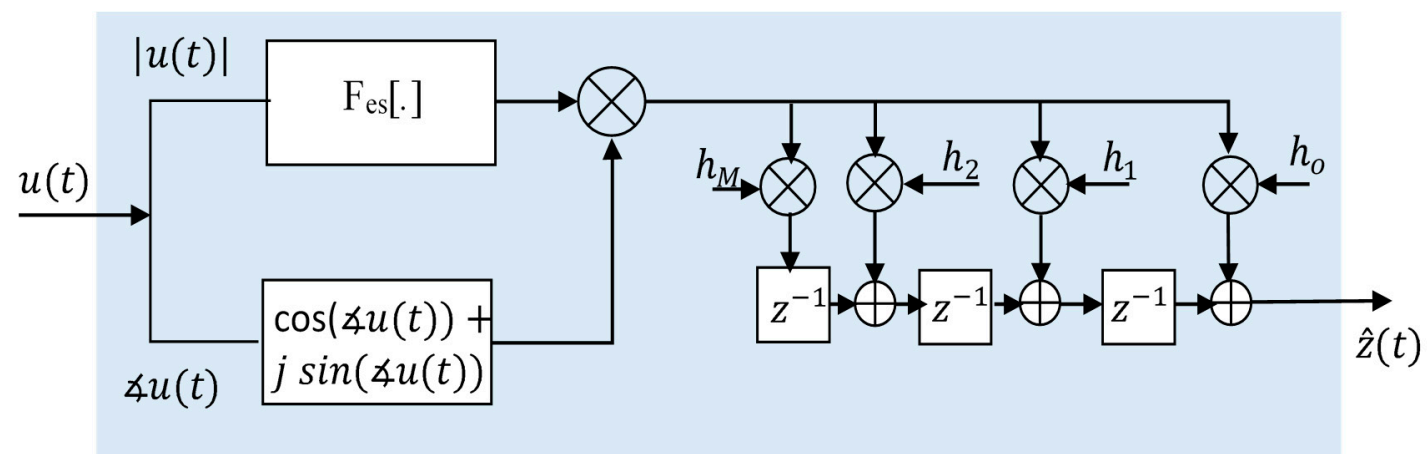

Figure 3. Hammerstein model structure for RF power amplifier using the enhanced Saleh amplitude-to-amplitude (AM/AM) model.

The FIR filter mimics the output signal dependency on the past samples of the input signal using a finite number of coefficients for controlling the memory depth in the model.

$$
\hat{z}(n)=\sum_{m=0}^{M} h(m) \mathrm{F}_{\mathrm{es}}[u(n-m)] e^{j \kappa u(n-m)}
$$

where $h(m)$ represents the filter impulse response, $M$ is the memory depth in the model, and $\hat{z}(n)$ is the output signal of the dynamic nonlinear model.

\section{Modeling of Digital Predistortion}

DPDs have become popular approaches for improving the linearity of RF PAs for a reliable and an efficient signal transmission [22,23]. The DPD implementation in the digital domain as in Figure 4 is practically more efficient than analog predistortion for compensating the nonlinear distortion in RF PAs. The DPD models based on Taylor polynomials are popular linearization techniques to compensate for a weak-nonlinear distortion in RF PAs [24]. 


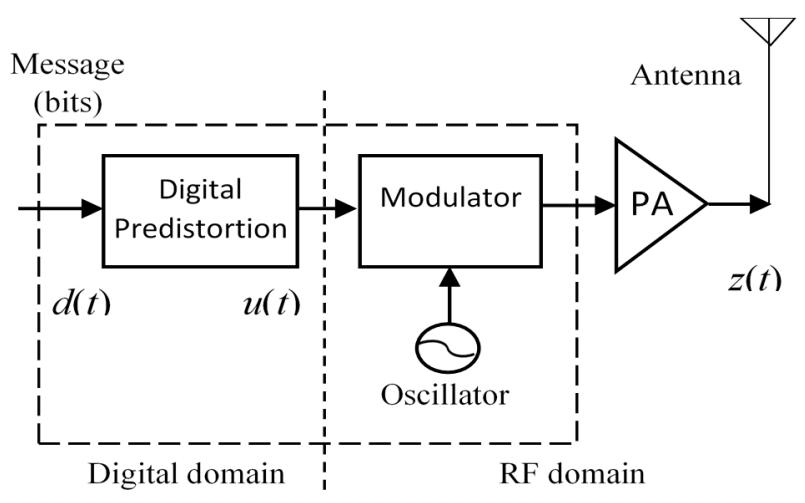

Figure 4. Simplified diagram of a digital predistortion transmitter system.

A complex structure of a DPD model is not desired in wireless communication, because this requires a complex and high-computational-cost hardware system. Hence, a predistortion model in this work is calculated directly from a simplified rational function of the enhanced Saleh model using fewer coefficients.

The proposed DPD model can be deployed for the PA of strong nonlinearity over a wide amplitude range. The mathematical inversion of the enhanced Saleh model is calculated as follows:

$$
u(t)=\mathrm{F}_{\mathrm{es}}^{-1}[d(t)]
$$

where $d(t)$ and $u(t)$ are the DPD model input and output magnitudes, respectively, as depicted on the simplified transmitter architecture in Figure $4 . \mathrm{F}_{\mathrm{es}}^{-1}[]$ is the proposed enhanced DPD model, which is calculated by exchanging the input and output variables of the enhanced Saleh model [11] $\mathrm{F}_{\mathrm{es}}[u(t)]$ in Equation (5) and simplifying the expression as:

$$
(d \beta-\lambda) u^{2}-\alpha u+d=0
$$

Finally, the DPD model is calculated by solving a quadratic formula in Equation (15) with respect to the variable $u$. An equation solution of a negative sign is used in this model, since the DPD input and output magnitudes are real and normalized in this work.

$$
\mathrm{F}_{\mathrm{es}}^{-1}[d]=\frac{\alpha-\sqrt{\alpha^{2}-4 \beta d^{2}+4 d \lambda}}{2(d \beta-\lambda)}
$$

Equation (16) represents the DPD model, which outputs a magnitude of the predistorted baseband signal. The maximum input amplitude of the DPD model is calculated to satisfy a positive real value inside the root term of Equation (16) as follows:

$$
\alpha^{2}+4 d_{\max } \lambda \geq 4 \beta d_{\max }^{2}
$$

The maximum amplitude of the DPD input $\left|d_{\max }\right|$ to satisfy Equation (17) is given by:

$$
\left|d_{\max }\right|=\frac{\lambda+\sqrt{\lambda^{2}+\beta \alpha^{2}}}{2 \beta}
$$

where $\left|d_{\max }\right|$ represents a clipping threshold of the DPD baseband input signal. Therefore, a clipping model in Equation (19) is required on the input of the DPD model to maintain the condition in Equation (17). 


$$
|\hat{d}(t)|=\left\{\begin{array}{cc}
|d(t)|, & \text { if }|d(t)|<\left|d_{\max }\right| \\
\left|d_{\max }\right|, & \text { if }|d(t)| \geq\left|d_{\max }\right|
\end{array}\right.
$$

where $|\hat{d}(t)|$ is a clipped amplitude version of the time domain signal $d(t)$. Figure 5 describes the characteristics of the clipping model in Equation (19) for the amplitude relationships between the input and output signals. This model forces the large amplitudes of the input signal to a constant threshold according to the value of $\left|d_{\max }\right|$, which is specified by the model parameters $(\alpha, \lambda, \beta)$. Equation (18) illustrates that the level magnitude of the clipping model is directly proportional to the power amplifier gain $(\alpha, \lambda)$ and inversely proportional to the parameter $\beta$. Although the model in Figure 5 is linear with unit gain in the amplitude range ( 0 to $\left.d_{\max }\right)$, the clipping model is another nonlinear function required on the input of the DPD model. The model clipping characteristics are depicted in Figure 6, which describe the variation in the input signal PAPR with respect to the clipping level $\left|d_{\max }\right|$ using an orthogonal frequency division multiplexing (OFDM) signal. The output amplitude becomes equal to a non-clipped amplitude for a large clipping level as illustrated.

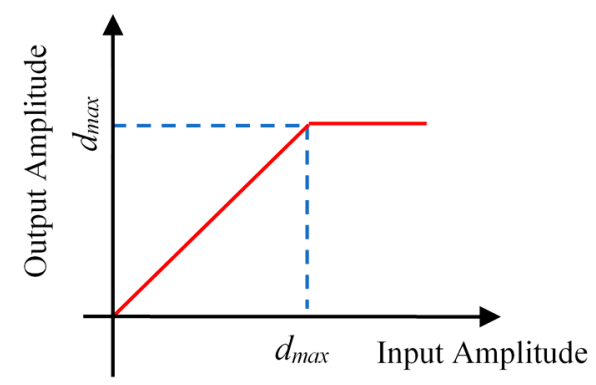

Figure 5. Signal amplitude limiter model required on the input of the digital predistortion (DPD) model.

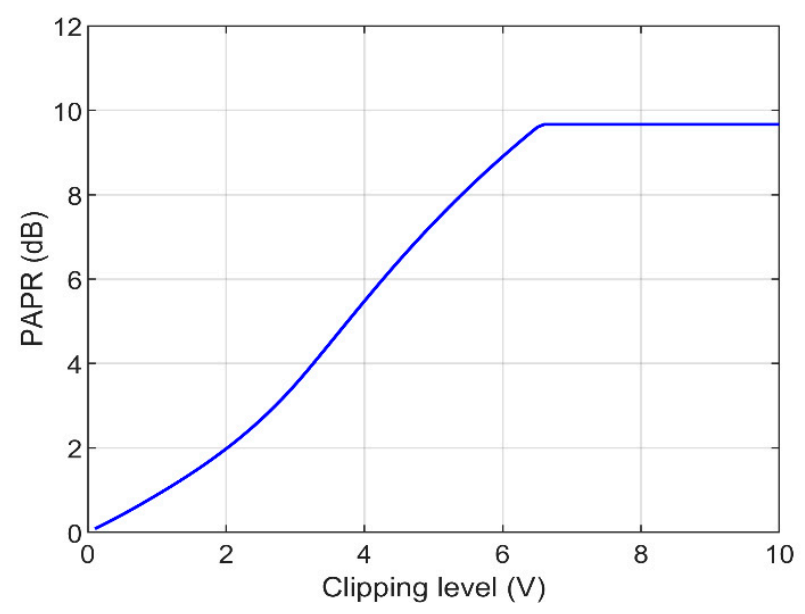

Figure 6. The model clipping level and the peak-to-average power ratio (PAPR) of the clipped signal.

The impact of the clipping nonlinearity on the distortion effects is not severe, because the large peaks typically occur with low probability. Therefore, different clipping approaches have been widely used in wireless communication to reduce the signal PAPR, and they are considered an important design aspect when operating RF PAs on OFDM signals. In addition, reducing the signal PAPR can improve the power efficiency in RF PAs, because the maximum power efficiency is obtained when operating RF PAs near the compression region [14]. Therefore, applying signal clipping in combination with DPD often leads to performance improvement in power efficiency and linearity [13,25-27]. 


\section{Evaluation Results}

Two different SSPA technologies, gallium arsenide (GaAs) (ZFL-1000LN from Mini-Circuits, Brooklyn, NY, USA) and gallium nitride (GaN) (ZX60-8008E from Mini-Circuits) devices under test (DUT) are used in this experiment for model evaluation and linearity enhancement of the DUT PA. The experiment set-up consists of a signal generator (E4438C from Keysight Technologies, Santa Rosa, CA, USA), a spectrum analyzer (RSA6120A from Tektronix, Beaverton, OR, USA), a high-power attenuator, and a computer (Dell computer with Intel Xeon CPU E3-1241 v3 @ 3.50 GHz and 16 GB of ram) with MATLAB (R2018a from MathWorks, Natick, MA, USA) as shown in Figure 7. A 64-QAM dual-band modulated OFDM signal was applied on the input of the SSPA, and the output signal is RF down-converted, demodulated, and acquired by the spectrum analyzer.

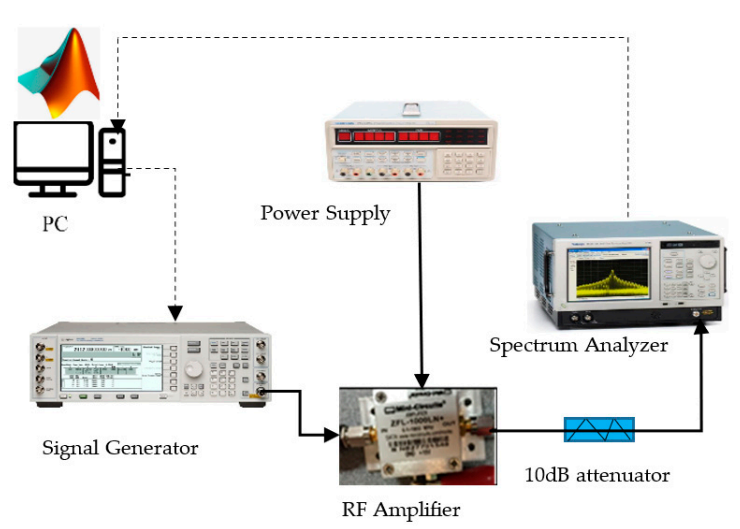

(a)

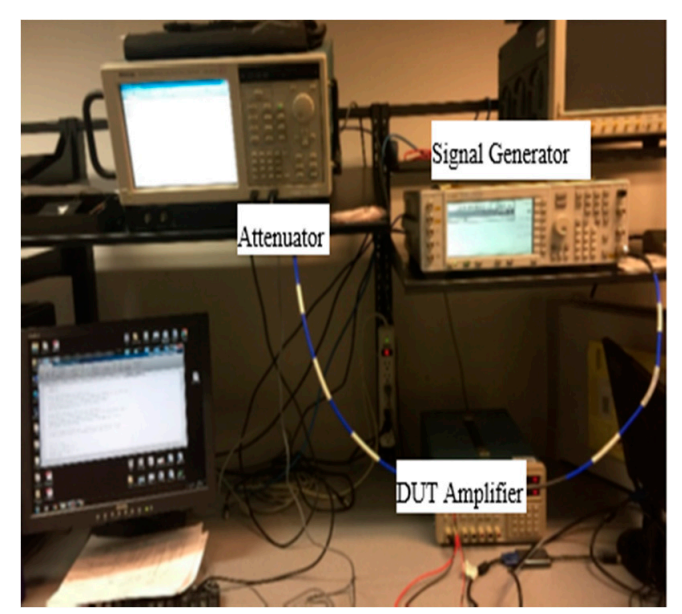

(b)

Figure 7. Experimental validation for modeling and digital predistortion of a solid-state power amplifier (SSPA). (a) Schematic diagram. (b) Photograph of the test set-up.

\subsection{SSPA Modeling Performance}

The AM/AM conversion of a GaAs RF PA is measured by sweeping the input amplitude of a two-tone signal from the signal generator at a $1 \mathrm{GHz}$ center frequency and a $10 \mathrm{KHz}$ tone spacing. The AM/AM nonlinear conversion of the SSPA measurements as well as the model estimation using both the Saleh and enhanced Saleh models is illustrated in Figure 8. The enhanced Saleh model shows improved curve-fitting results in the linear and compression regions compared to the original Saleh model. In addition, the residual square errors between the measured and model conversions $\left(\left|z_{\text {meas }}\right|-\right.$ $\left.\left|z_{\text {mode }}\right|\right)^{2}$ clearly show lower fluctuation errors and accuracy improvement of the enhanced Saleh model with respect to the input amplitude.

The model evaluation for predicting the nonlinear distortion in a frequency domain is shown in Figure 9, using the power spectrum density of a dual-band long-term evolution (LTE) signal. Table 1 presents the obtained accuracy improvement in the enhanced Saleh model using the normalized mean square-errors (NMSE) based on GaAs and GaN DUT PA technologies. These results show a model improvement of around 4dB NMSE compared to the original Saleh model. In addition, the enhanced model achieved better model accuracy compared to the fifth-order Taylor model. 


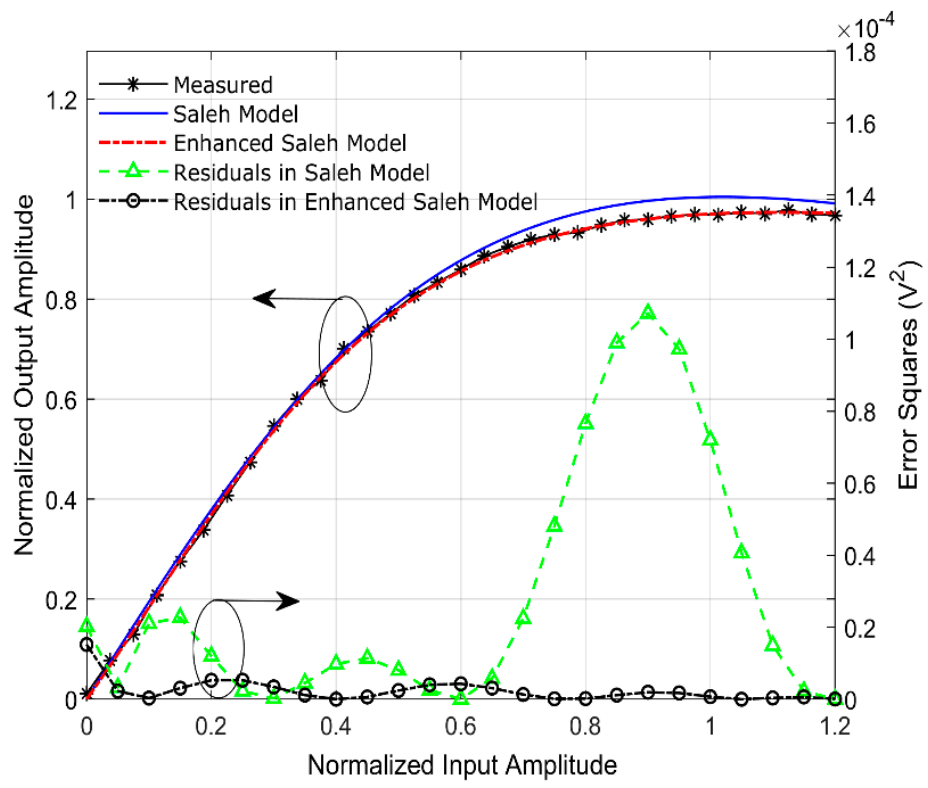

Figure 8. Measured and modeled static AM/AM results of the SSPA using a two-tone test. The square residual errors of the Saleh model and enhanced Saleh model are overlaid with respect to the input amplitude.

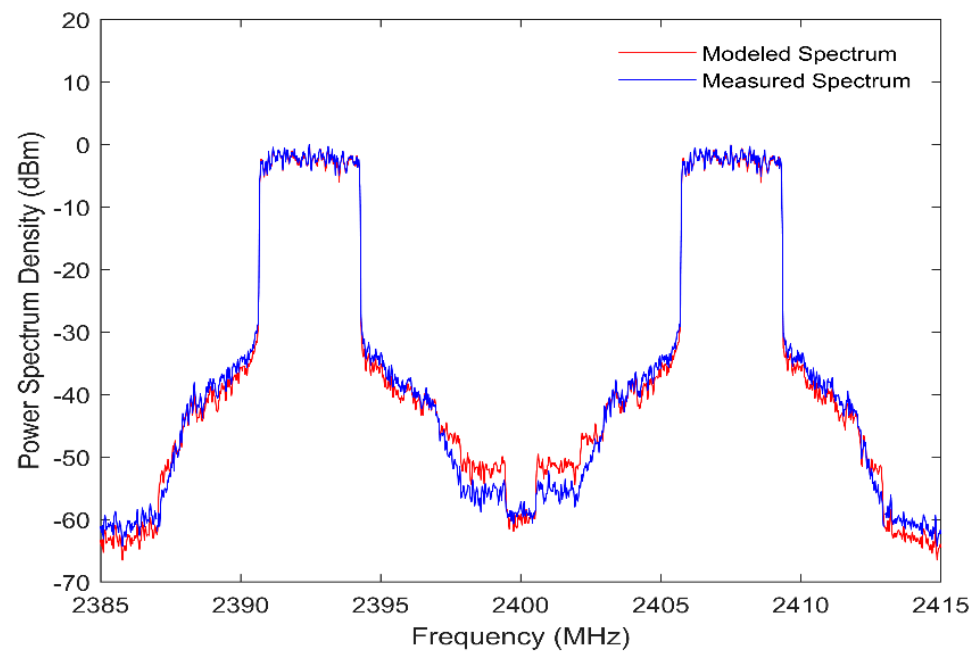

Figure 9. Measured and modeled output spectrum of the solid-state power amplifier using a dual-band LTE signal.

Table 1. Accuracy comparison in normalized mean square-errors (NMSE) of the model evaluation using gallium arsenide ( $\mathrm{GaAs})$ and gallium nitride $(\mathrm{GaN})$ technologies.

\begin{tabular}{ccccc}
\hline \multirow{2}{*}{ Model } & \multirow{2}{*}{ Number of Coeff. } & $\begin{array}{c}\text { Intermodulation Distortion } \\
\text { (IMD) }\end{array}$ & \multicolumn{2}{c}{ NMSE (dB) } \\
\cline { 3 - 5 } & 2 & GaAs PA & GaN PA \\
\hline Saleh & 3 & odd terms & -27.12 & -28.54 \\
\hline Enhanced Saleh & 5 & odd and even terms & -31.37 & -32.71 \\
\hline 5th order Polynomial & & odd and even terms & -22.16 & -21.47 \\
\hline
\end{tabular}

The overall accuracy in NMSE of the enhanced Saleh model in cascade with FIR filter is depicted in Figure 10, with respect to the FIR filter number of coefficients (i.e., memory depth). This shows the obtained optimal model accuracy and the required filter number of coefficients to quantify the 
memory effect of the SSPA. The FIR filter in this modeling approach reaches the optimal accuracy at a memory depth around 15 for practical implementation, and the accuracy in NMSE changes slightly and becomes almost flat after in the higher order memory depth as illustrated in Figure 10. The dynamic enhanced model achieves a NMSE below $-47 \mathrm{~dB}$.

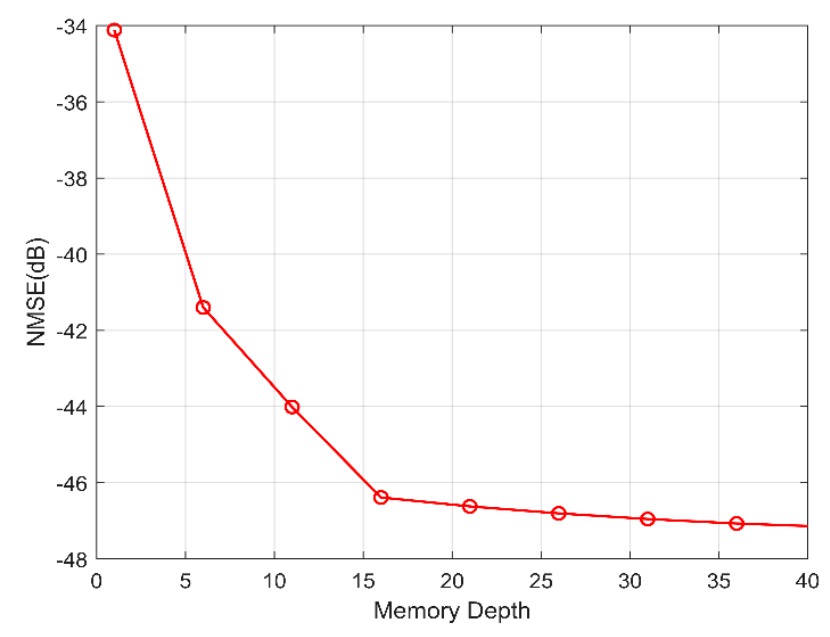

Figure 10. The model accuracy in normalized mean square-error (NMSE) of the dynamic enhanced Saleh model in terms of the memory depth of the finite-impulse response (FIR) filter.

\subsection{Results of Digital Predistortion}

The static AM/AM conversion causes most the nonlinear distortion in RF PAs. Therefore, the efficiency of the DPD to compensate for the AM/AM distortion is a significant factor in linearizing the nonlinearity in communication systems. The DPD model based on the enhanced Saleh model is evaluated in the time and frequency domains using a dual-band LTE signal. The AM/AM conversion of the DPD model exhibits significant gain expansion characteristics over a wide range of the PA input amplitude as shown in Figure 11. In addition, the DPD model compensated for the gain compression behavior of the SSPA and improved the balance between in-phase and quadrature-phase in the signal constellation diagram, as shown in Figure 12.

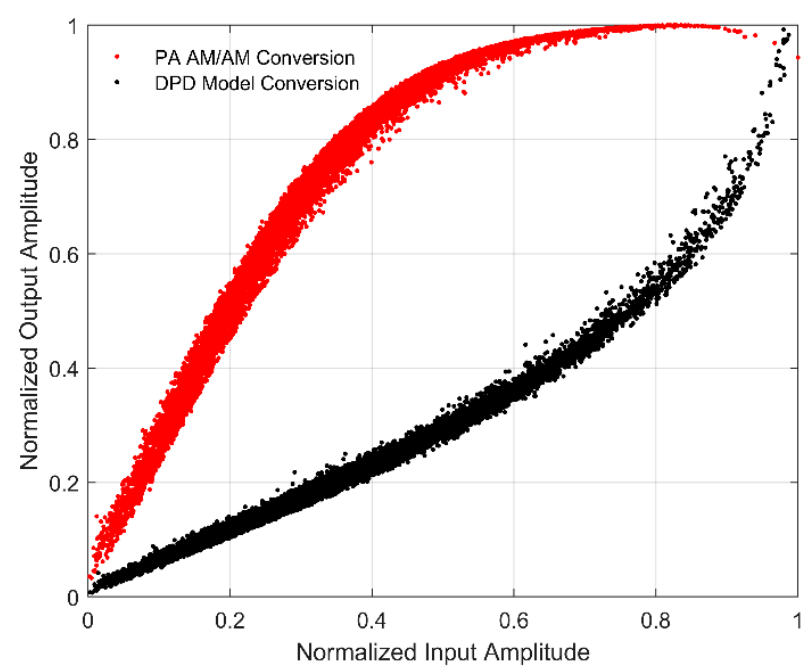

Figure 11. The dynamic AM/AM conversion of the RF power amplifier and DPD model conversions based inverting the enhanced Saleh model. 


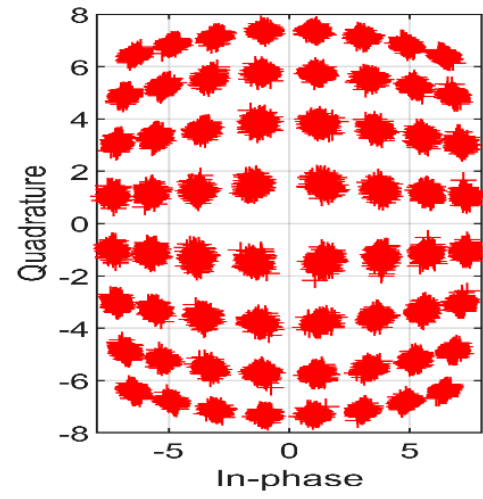

(a)

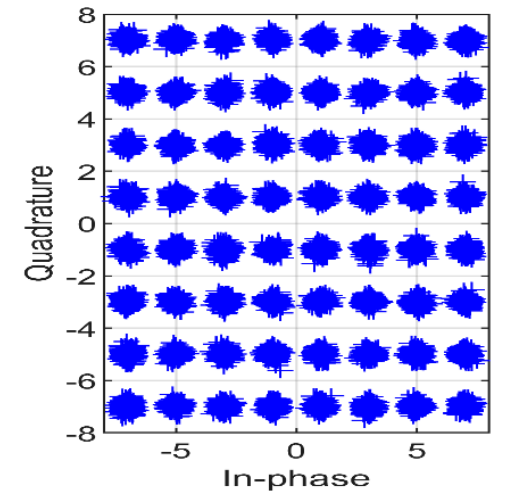

(b)

Figure 12. Constellation diagram of a 64-QAM baseband output signal of the RF power amplifier, (a) without DPD, and (b) with DPD.

The linearization capability of the DPD model in a frequency domain shows significant mitigation of the spectrum regrowth in the adjacent channels of the dual-band spectrum as depicted in Figure 13. The numerical evaluation results of this linearization approach is depicted in Table 2 using the adjacent channel power ratio (ACPR) and error vector magnitude (EVM) for GaAs and GaN SSPA. Finally, the obtained linearization performance of the enhanced DPD model achieved an adequate improvement in time and frequency domains.

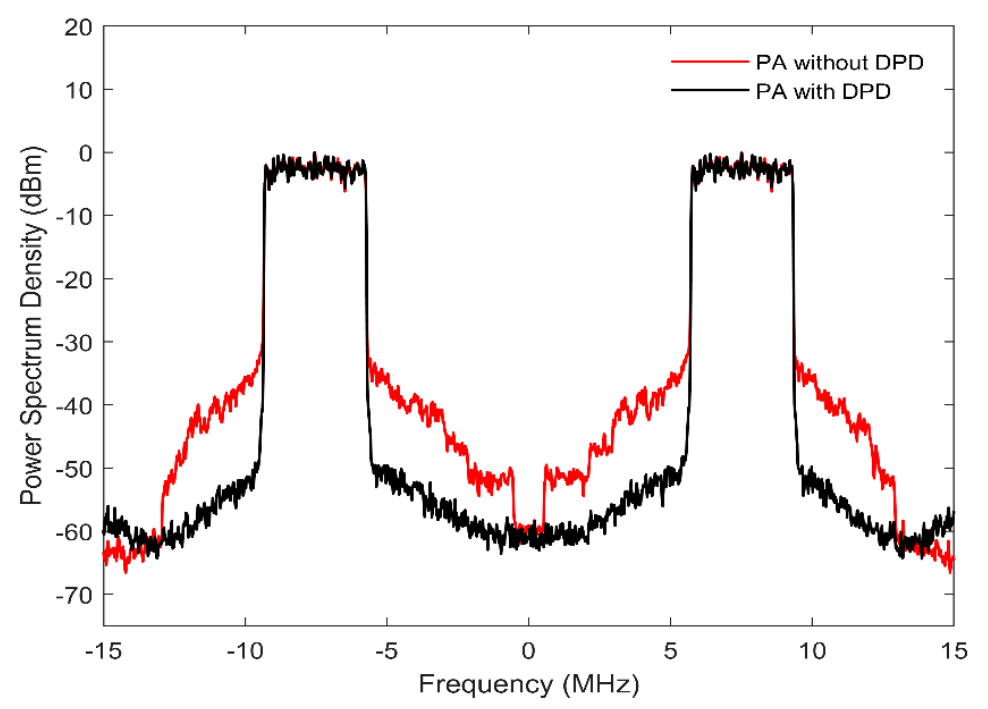

Figure 13. Spectrum of a dual-band LTE output signal without DPD in the red trace and with DPD in the black trace.

Table 2. DPD model performance in adjacent channel power ratio (ACPR) and error vector magnitude (EVM) for linearizing both GaAs and GaN power amplifiers (PAs).

\begin{tabular}{ccccc}
\hline Evaluation Measure & without DPD & with DPD & without DPD & with DPD \\
\hline $\begin{array}{c}\text { ACPR in Upper } \\
\text { band/Lower band (dBc) }\end{array}$ & $-38.73 /-39.35$ & $-52.10 /-52.81$ & $-41.86 /-42.55$ & $-56.13 /-57.41$ \\
\hline EVM (\%) & 9.12 & 1.86 & 8.76 & 1.54 \\
\hline
\end{tabular}




\section{Conclusions}

This paper analyzed the Saleh model mathematically using polynomial expansion for modeling the odd-order IMD and described the Saleh weakness for modeling SSPAs. Therefore, a new model enhancement was proposed to improve the model accuracy for the Saleh AM/AM model when deployed for SSPA technologies in communication systems. In addition, the polynomial expansion of the enhanced Saleh model consists of both odd-order and even-order nonlinear terms for characterizing the PA nonlinearities in the AM/AM conversion. The Hammerstein-based model structure was adopted to quantify the dynamic effect in the AM/AM conversion. A least-squares approach was employed for calculating all the parameters of the enhanced Saleh model. The evaluation results showed an accuracy improvement of the enhanced Saleh model to reflect the measured AM/AM characteristics of SSPAs over a wide range of the PA input magnitude. A linearization approach using DPD was developed and evaluated for mitigating the nonlinear distortion in SSPAs.

Author Contributions: Conceptualization, H.A.; Methodology, H.A. and F.L.; Software, H.A.; Supervision, F.L.; Writing - original draft, H.A.; Writing_review \& editing, H.A. and F.L. All authors have read and agreed to the published version of the manuscript.

Funding: Publication of this article is funded by Portland State University's open access fund.

Acknowledgments: The authors would like to acknowledge James Morris from the Department of electrical and computer engineering at Portland State University for contributions on proofreading this article.

Conflicts of Interest: The authors declare no conflict of interest.

\section{References}

1. Popovic, Z. Amping Up the PA for 5G: Efficient GaN Power Amplifiers with Dynamic Supplies. IEEE Microw. Mag. 2017, 18, 137-149. [CrossRef]

2. Singh, S.K.; Suthar, L.; Singh, R.; Kumar, A. GaN Based S-Band 500W Solid State Power Amplifier (SSPA) Module for Troposcatter Communications. In Proceedings of the 2019 IEEE MTT-S International Microwave and RF Conference (IMARC), Mumbai, India, 13-15 December 2019; pp. 1-4.

3. Davis, C.; Hawkins, J.; Einolf, C. Solid state DTV transmitters. IEEE Trans. Broadcast. 1997, 43, $252-260$. [CrossRef]

4. Hu, S.; Xu, K.; Hu, Y.; Wu, K.; Qian, J.; Gao, Y.; Song, D.; Wang, Z.; Zhou, B.; Cai, Z.; et al. Design of a compact chip filter with two transmission zeros using $0.35 \mu \mathrm{m}$ GaAs HBT. Microelectron. J. 2020, 95, 104661. [CrossRef]

5. Sun, J.; Shi, W.; Yang, Z.; Yang, J.; Gui, G. Behavioral Modeling and Linearization of Wideband RF Power Amplifiers Using BiLSTM Networks for 5G Wireless Systems. IEEE Trans. Veh. Technol. 2019, 68, 10348-10356. [CrossRef]

6. Ghannouchi, F.; Hammi, O.; Helaoui, M. Behavioral Modeling and Predistortion of Wideband Wireless Transmitters; Wiley: Hoboken, NJ, USA, 2015; pp. 43-63.

7. Cann, A.J. Improved nonlinearity model with variable knee sharpness. IEEE Trans. Aerosp. Electron. Syst. 2012, 48, 3637-3646. [CrossRef]

8. Saleh, A. Frequency-Independent and Frequency-Dependent Nonlinear Models of TWT Amplifiers. IEEE Trans. Commun. 1981, 29, 1715-1720. [CrossRef]

9. O'Droma, M.; Meza, S.; Lei, Y. New modified Saleh models for memoryless nonlinear power amplifier behavioural modelling. IEEE Commun. Lett. 2009, 13, 399-401. [CrossRef]

10. Ochiai, H. An Analysis of Band-limited Communication Systems from Amplifier Efficiency and Distortion Perspective. IEEE Trans. Commun. 2013, 61, 1460-1472. [CrossRef]

11. Nguyen, T.; Yoh, J.; Lee, C.; Tran, H.; Johnson, D. Modeling of hpa and hpa linearization through a predistorter: Global broadcasting service applications. IEEE Trans. Broadcast. 2003, 49, 132-141. [CrossRef]

12. Al Kanan, H.; Yang, X.; Li, F. Improved estimation for Saleh model and predistortion of power amplifiers using 1-dB compression point. J. Eng. 2020, 2020, 13-18. [CrossRef]

13. Van Nee, R.; Prasad, R. OFDM for Wireless Multimedia Communications; Artech House: Norwood, MA, USA, 2000.

14. Cripps, S.C. Advanced Techniques in RF Power Amplifiers Design; Artech House: Nonvood, MA, USA, 2002. 
15. Al-Kanan, H.; Yang, X.; Li, F. Saleh model and digital predistortion for power amplifiers in wireless communications using the third-order intercept point. J. Electron. Test. 2019, 35, 359-365. [CrossRef]

16. $\mathrm{Xu}, \mathrm{K}$. Integrated Silicon Directly Modulated Light Source Using p-Well in Standard CMOS Technology. IEEE Sens. J. 2016, 16, 6184-6191. [CrossRef]

17. Al-kanan, H. Power Efficiency Enhancement and Linearization Techniques for Power Amplifiers in Wireless Communications. Ph.D. Dissertation, Portland State University, Portland, OR, USA, 2020.

18. Singh, A.; Scharer, J.; Booske, J.; Wohlbier, J. Second- and Third-Order Signal Predistortion for Nonlinear Distortion Suppression in a TWT. IEEE Trans. Electron Devices 2005, 52, 709-717. [CrossRef]

19. Ding, L.; Zhou, G. Effects of Even-Order Nonlinear Terms on Power Amplifier Modeling and Predistortion Linearization. IEEE Trans. Veh. Technol. 2004, 53, 156-162. [CrossRef]

20. Moon, J.; Kim, B. Enhanced Hammerstein Behavioral Model for Broadband Wireless Transmitters. IEEE Trans. Microw. Theory Tech. 2011, 59, 924-933. [CrossRef]

21. Al-Kanan, H.; Li, F.; Tafuri, F.F. Extended Saleh model for behavioral modeling of envelope tracking power amplifiers. In Proceedings of the 2017 IEEE 18th Wireless and Microwave Technology Conference (WAMICON), Cocoa Beach, FL, USA, 24-25 April 2017; pp. 1-4.

22. Ren, J. A New Digital Predistortion Algorithms Scheme of Feedback FIR Cross-Term Memory Polynomial Model for Short-Wave Power Amplifier. IEEE Access 2020, 8, 38327-38332. [CrossRef]

23. Jang, K.; Ryu, H.-G.; Ryu, S.B.; Lee, S.G. Spectrum characteristics and predistortion gain in the nonlinear high power amplifier (HPA). In Proceedings of the 2017 International Conference on Information and Communication Technology Convergence (ICTC), Jeju Island, Korea, 18-20 October 2017; pp. 931-934.

24. Zhou, G. Analysis of spectral regrowth of weakly nonlinear power amplifiers. IEEE Commun. Lett. 2000, 4, 357-359. [CrossRef]

25. Wang, J.; Xu, Y.; Zhu, X. Digital predistorted inverse class-F GaN PA with novel PAPR reduction technique. In Proceedings of the 2011 IEEE MTT-S International Microwave Symposium, Baltimore, MD, USA, 5-10 June 2011.

26. Chen, S. An Efficient Predistorter Design for Compensating Nonlinear Memory High Power Amplifiers. IEEE Trans. Broadcast. 2011, 57, 856-865. [CrossRef]

27. Wang, Y.; Akansu, A.N. Low-complexity peak-to-average power ratio reduction method for orthogonal frequency-division multiplexing communications. IET Commun. 2015, 9, 2153-2159. [CrossRef]

Publisher's Note: MDPI stays neutral with regard to jurisdictional claims in published maps and institutional affiliations.

(C) 2020 by the authors. Licensee MDPI, Basel, Switzerland. This article is an open access article distributed under the terms and conditions of the Creative Commons Attribution (CC BY) license (http://creativecommons.org/licenses/by/4.0/). 